En ce qui concerne la distribution directe des médicaments par le médecin (DMA), la situation reste ouverte et plusieurs questions doivent encore être tirées au clair, même et surtout après la dernière publication de l'OFAS à ce sujet. Cela n'a rien à faire avec TarMed lui-même mais beaucoup avec les dispositions légales attendues au niveau fédéral. Cela est à son tour lié à la nouvelle loi sur les agents thérapeutiques autour de laquelle le Parlement s'affaire encore, en des affrontements toujours plus virulents. Il est dans ces conditions plus que douteux que ces textes législatifs soient connus ou même entrés en vigueur d'ici à l'introduction des nouveaux tarifs.

Dans cette situation, la FMH soutient à titre de condition sine qua non la position selon laquelle la DMA doit faire partie intégrante de la convention sur la neutralité des coûts en ce sens que d'éventuelles diminutions de revenu dues à la DMA devront être compensées par des augmentations de la VdP sur les prestations médicales.

Nous ne sommes pas davantage au clair en ce qui concerne la nouvelle Liste des analyses pour laquelle nous sommes dans le flou aussi bien en ce qui concerne son contenu que le moment où elle va être édictée. La FMH soutient ici mutatis mutandis la même position que pour la DMA, une position qui vaut aussi pour la reprise d'autres ouvres tarifaires par le TarMed, par ex. le tarif de physiothérapie.
Les auteurs d'offres de cours de formation TarMed les plus divers se bousculent actuellement sur un marché promettant à n'en pas douter des bénéfices juteux. Il leur manque le plus souvent des connaissances solides sur la structure tarifaire, mais surtout sur l'arrière-plan de ces connaissances, si important pour dispenser des cours de formation. Du côté de la FMH nous ne pouvons recommander que des cours de formation organisés par la FMH, resp. les sociétés cantonales de médecine et les sociétés de discipline médicale en collaboration avec la FMH, ainsi que les cours de la Caisse des médecins.

Le paysage de la politique de santé dans notre pays est très labile; cela se fera également sentir sur l'introduction des tarifs TarMed (et au delà). Il faut compter d'être confronté à de nouvelles situations, voire à des surprises. La ronde des cotisations 2000 à laquelle nous assistons actuellement va sûrement augmenter la pression sur le TarMed et cela d'autant plus qu'un nombre croissant de responsables politiques de ce pays prennent conscience de ce que le système suisse de santé publique souffre d'absence de pilotage et de concepts.

1 Deschenaux F-X. Rapport sur la session ordinaire de la Chambre médicale suisse des 21 et 22 juin 2000 à Berne. Bull Med Suisses 2000;81(31):1680-90.

\title{
Ordner Qualitätsmanagement im Gesundheitswesen
}

Während der akademischen Jahre 1998/1999 und 1999/2000 hat die Verbindung der Schweizer Ärztinnen und Ärzte zwei Serien von Seminaren zum Thema Qualitätsmanagement (QM) durchgeführt.

Zwei Lernziele standen dabei im Vordergrund:

a) Vermehrung des Wissens betreffend Qualitätsmanagement

b) Vorbereitung zur selbständigen Durchführung von qualitätsfördernden Projekten

Eine Auswahl der Unterlagen erscheint jetzt in Form eines Ordners (nur in deutscher Sprache, 264 Seiten, Verzeichnis der Kapitel liegt bei).

Aufgrund der im Verlauf der Seminare gesammelten Erfahrungen wird im akademischen Jahr 2000/ 2001 eine neu gestaltete Reihe von QM-Seminaren durch das College-M und die FMH durchgeführt (SÄZ, 2000:81. Nr. 21. Seiten 1097-1099).

Die in diesem Ordner präsentierten Texte können nicht nur für die Teilnehmerinnen und Teilnehmer der neuen Seminarreihe von Nutzen sein, sondern für alle Personen, die sich mit der QM-Problematik beschäftigen.
Kapitelübersicht

- Gesetzliche Grundlagen und QS/QF-Projekte

- Methoden

- Grundlagen der Gesundheitsökonomie

- Leitlinien

- Angemessenheit medizinischer Leistungen

- QM-Projekte

- Patienten und Qualität

- Beispiele spezieller QM-Programme

- Akkreditierung und Zertifizierung

- Weiterführende Literatur und Internetadressen

Bestellungen:

Der FMH-Ordner "Qualitätsmanagement im Gesundheitswesen" kann zum Preis von Fr. 55.- (inkl. Versandkosten und MwSt) bestellt werden.

FMH

Daniel Rodriguez

Elfenstrasse 18

Postfach 293

3000 Bern 16

Fax-Nr. 031/359 1112

E-Mail: fmh@hin.ch 